\title{
Firing Properties of Head Direction Cells in the Rat Anterior Thalamic Nucleus: Dependence on Vestibular Input
}

\author{
Robert W. Stackman and Jeffrey S. Taube \\ Department of Psychology, Dartmouth College, Hanover, New Hampshire 03755
}

\begin{abstract}
Vestibular information influences spatial orientation and navigation in laboratory animals and humans. Neurons within the rat anterior thalamus encode the directional heading of the animal in absolute space. These neurons, referred to as head direction $(\mathrm{HD})$ cells, fire selectively when the rat points its head in a specific direction in the horizontal plane with respect to the external laboratory reference frame. HD cells are thought to represent an essential component of a neural network that processes allocentric spatial information. The functional properties of HD cells may be dependent on vestibular input. Here, anterior thalamic HD cells were recorded before and after sodium arsanilate-induced vestibular system lesion. Vestibular lesions abolished the directional firing properties of HD cells. The time course of disruption in the directional firing properties paralleled the loss of vestibular function. Arsanilate-treated rats
\end{abstract}

exhibited only minor changes in locomotor behavior, which were unlikely to account for the loss of direction-specific firing. Vestibular lesions also disrupted the influence of angular head velocity on anterior thalamic single-unit firing rates. Finally, a subset of anterior thalamic neurons recorded from vestibularlesioned rats exhibited a pattern of intermittent firing bursts that were distinctly unrelated to HD. This novel anterior thalamic firing pattern has not been encountered in any vestibular-intact rat. These data suggest that: (1) the neural code for directional bearing is critically dependent on vestibular information; and (2) this loss of HD cell information may represent a neurobiological mechanism to account for the orientation and navigational deficits observed after vestibular dysfunction.

Key words: head direction cells; navigation; angular head velocity; spatial orientation; thalamus; vestibular dysfunction
Accurate spatial orientation and navigation require the estimation of current location and direction in space from two distinct neural systems; one system uses external sensory cues or landmarks, and the other system uses internally generated signals (e.g., vestibular, proprioceptive, and motor efference copy) for path integration (Barlow, 1964; Gallistel, 1990; McNaughton et al., 1995; Berthoz et al., 1995). For humans, the sense of direction is arguably derived from an integrated multimodal neural system encoding allocentric space. In the absence of familiar landmarks, internal cues are thought to provide sufficient sensory information to support accurate spatial navigation through path integration. Vestibular-deficient humans exhibit only minor navigational impairments because of an enhanced reliance on visual landmarks. However, in the dark or when blindfolded, vestibular impaired humans show marked deficits in spatial navigation (Beritoff, 1965; Heimbrand et al., 1991; Pozzo et al., 1991; Brookes et al., 1993). Animals are known to use vestibular information to guide spatial navigation (Mittelstaedt and Mittelstaedt, 1980), and vestibular lesions disrupt performance on spatial navigation tasks (Potegal et al., 1977; Miller et al., 1983; Matthews et al., 1989; Ossenkopp and Hargreaves, 1993).

Navigation requires the encoding and maintenance of representations regarding the spatial relationship of the organism to its environment. Neurons found in the rat postsubiculum $(\mathrm{PoS})$

Received Oct. 18, 1996; revised Feb. 28, 1997; accepted March 24, 1997.

This work was supported in part by National Institute on Deafness and Other Communication Disorders Grant DC00236 (R.W.S.) and National Institute of Mental Health Grants MH48924 and MH01286 (J.S.T.). We thank P. Dudchenko and J. Goodridge for technical assistance, M. Glickstein for valuable editorial comments, K-P. Ossenkopp for advice regarding the use of sodium arsanilate, and Abbott Laboratories for generously supplying the compound.

Correspondence should be addressed to Dr. Jeffrey S. Taube, Department of Psychology, Dartmouth College, 6207 Gerry Hall, Hanover, NH 03755.

Copyright (C) 1997 Society for Neuroscience 0270-6474/97/174349-10\$05.00/0
(Taube et al., 1990a,b) and anterodorsal thalamic nucleus (ATN) (Taube, 1995) discharge as a function of the directional heading of the animal, independent of its location or other ongoing behaviors. Each of these head direction (HD) cells fires maximally (peak firing rate) at one preferred direction. Although salient visual cues exert control over the preferred firing direction of the HD cell (Goodridge and Taube, 1995), cell discharge is maintained in the absence of landmarks, indicating that alternate sources of information, possibly vestibular, also influence HD cell firing (Goodridge and Taube, 1995; Taube and Burton, 1995). It is thought that in the absence of visual landmarks, computation of current directional heading is accomplished by an integration of ongoing head movements (McNaughton et al., 1991). An angular velocity signal relayed from the vestibular system to the hippocampal formation may be necessary for encoding a representation of spatial relationships among environmental cues. Indeed, vestibular stimulation influences hippocampal place cell activity (Sharp et al., 1995; Wiener et al., 1995) and anterior thalamic HD cells in the rat (Blair and Sharp, 1996). Taken together, the vestibular system seems to be essential for accurate spatial navigation and for the neural representation of head direction.

The present experiment examined the discharge properties of ATN HD cells before and after vestibular apparatus lesions. Vestibular lesions were induced by bilateral intratympanic administration of sodium arsanilate (30 mg/side) (Horn et al., 1981; Ossenkopp et al., 1990). Single-unit recordings of ATN HD cells demonstrated that sodium arsanilate disrupted the directional firing patterns of these neurons. The lesion-induced loss of directional firing was coincident with the expression of overt behaviors indicative of vestibular dysfunction. This result provides direct neurobiological evidence of the role of vestibular signals in the directional sense. 


\section{MATERIALS AND METHODS}

Subjects and training. Subjects were 11 female Long-Evans rats, weighing $250-300 \mathrm{gm}$ at the beginning of the experiment. Rats were maintained on a food-restricted diet (15-20 gm/d) and housed separately in suspended wire mesh cages. Tap water was available ad libitum. All training, unit screening, and recording occurred during sessions in which the rats foraged for food pellets in a gray cylindrical apparatus $(76 \mathrm{~cm}$ diameter $51 \mathrm{~cm}$ high). A black floor-to-ceiling curtain enclosure $(2 \mathrm{~m}$ diameter $)$ surrounded the cylinder, and four uniformly arranged overhead DC lamps provided sufficient illumination. A color video camera (XC-711; Sony, Tokyo, Japan) was centered above the cylinder $3 \mathrm{~m}$ from the floor surface. The cylinder was placed on a sheet of gray photographic backdrop paper. A white cue card attached to the inside wall of the cylinder, occupying approximately $100^{\circ}$ of arc, served as a visual landmark. Rats received at least five training trials $(1 \mathrm{trial} / \mathrm{d})$ during which food pellets ( $20 \mathrm{mg}$; PJ Noyes, Lancaster, $\mathrm{NH}$ ) were thrown randomly into the cylinder. By the completion of training, rats engaged in nearly continuous food pellet search behavior over the entire floor of the cylinder.

Electrode implantation. Electrode construction and implantation techniques were similar to those described previously (Taube, 1995). Briefly, each electrode array consisted of a bundle of $10,25 \mu \mathrm{m}$ diameter, nichrome wires (California Fine Wire Co., Grover City, CA) insulated except at the tips. The wire bundle was passed through a 26 gauge stainless steel cannula (Small Parts, Miami, FL) and attached to a modified 11 pin Augat connector. The electrode array could be advanced in the dorsoventral plane through the use of three screws attached to the acrylic base of the electrode (Kubie, 1984). After habituation to the cylindrical apparatus and the expression of adequate food pellet foraging behavior, each rat was anesthetized with sodium pentobarbital $(45 \mathrm{mg}$ ) $\mathrm{kg}$, i.p.) and stereotaxically implanted with an electrode array directed at the ATN. Electrode coordinates were as follows: from bregma: anterior/ posterior, $-1.4 \mathrm{~mm}$; medial/lateral $+1.3 \mathrm{~mm}$; and ventral, $4.0 \mathrm{~mm}$ from the cortical surface (Paxinos and Watson, 1986). All procedures were conducted according to an institutionally approved animal care protocol. All surgical procedures were conducted under sterile conditions, and the rats were allowed a 1 week postoperative recovery interval before singleunit screening commenced.

Isolation and recording of ATN single-unit activity. After recovery from surgery, the activity from each electrode wire was assessed during daily unit screening sessions while the rat foraged for food pellets in the cylinder. Each rat was transported into the screening area from the animal colony room in a corrugated cardboard enclosure $(\sim 30 \times \sim 30 \mathrm{~cm}$ box). The cardboard box was placed on the floor inside the curtained enclosure next to the cylinder. A recording cable was attached to the implanted electrode while the rat was held gently in a towel. The rat was then released into the cylinder apparatus, at a start position that varied daily in a pseudorandom manner. Unit activity was recorded using procedures similar to those described previously (Taube et al., 1990a; Taube, 1995). Briefly, electrical signals were passed through a field effect transistor (FET) (one FET per electrode) in a source-follower configuration and through an overhead communator (Biela Development), amplified (Grass Instruments P5 series), bandpass-filtered $(300-10,000 \mathrm{~Hz}, 3 \mathrm{~dB}$ octave; Peavey Electronics PME8), and passed through a series of window discriminators (BAK Electronics DDIS-1). The resultant signal was then displayed on an oscilloscope (Tektronix 2214). Electrode activity was monitored while observing the rat's behavior on a video monitor with a camera mounted $3 \mathrm{~m}$ above the cylinder floor. If HD cell activity was not found, the electrodes were advanced 25-50 $\mu \mathrm{m}$ further into the ATN, and the activity was monitored on the electrodes again the next day. Screening for cells occurred over the course of several weeks.

When an HD cell waveform could be sufficiently isolated from the background electrical noise, two light-emitting diodes (LEDs; a red LED positioned over the rat's snout and a green LED positioned over its back) were added to the recording cable. The $x$ and $y$ coordinates of the LEDs were monitored at $60 \mathrm{~Hz}$ by a video tracking system (Eberle Electronics). During 8 min recording sessions the LED coordinates and cell activity were sampled at $60 \mathrm{~Hz}$ and acquired by a data acquisition board (National Instruments DIO-96) in a personal computer (Apple Macintosh Quadra 840AV). Data were stored for subsequent off-line analyses using LabView 2.2 (National Instruments) software programs. The location of the rat was defined as the point $25 \%$ of the distance from the red LED along the line between the two LEDs; this point corresponded to the position of the rat's head.

Testing of vestibular function and vestibular lesion. Prelesion vestibular function was assessed using a contact-righting test (Chen et al., 1989).
The contact-righting test is sensitive to vestibular dysfunction induced both surgically and by intratympanic injection of sodium arsanilate (Chen et al., 1986; Shoham et al., 1989) and was chosen to assess labyrinthine righting while limiting disturbance of the ATN microdrive and electrode arrays. The contact-righting test required placing the rat supine on a tabletop surface and bringing a Plexiglas surface into gentle contact with the ventral surface of the rat's feet. Intact rats will rapidly right themselves on making contact with the second surface, whereas vestibularlesioned rats will remain in the supine position. After testing of vestibular function, rats were anesthetized with Brevital $(50 \mathrm{mg} / \mathrm{kg})$, a 26 gauge injection needle was advanced through the tympanic membrane, and 0.1 $\mathrm{ml}$ of sodium arsanilate $(300 \mathrm{mg} / \mathrm{ml}$ dissolved in sterile $0.9 \%$ saline; Abbott Laboratories, Abbott Park, IL) was infused bilaterally over approximately $5 \mathrm{sec}$. On completion of the infusion the ear canal was packed with Gelfoam. Sodium arsanilate treatment produces a degeneration of the neuroepithelium of the vestibular cristae ampullares, maculae utriculi, and the cochlea after systemic (Anniko and Wersäll, 1977) or intratympanic injection (Kaufman et al., 1992). This degeneration is believed to arise from a disruption of osmolality in the vestibular apparatus and leads to the destruction of hair cells (Anniko and Wersäll, 1977). Furthermore, histological analyses have revealed that intratympanic injection of sodium arsanilate causes a degeneration of the vestibular nerve in the brainstem (Chen et al., 1986). The extent to which sodium arsanilate treatment leads to the degeneration of brainstem (e.g., vestibular and cochlear) and cerebellar nuclei has not been fully assessed. However, complete recovery of the spontaneous activity of central vestibular neurons has been reported 7 days after unilateral surgical labyrinthectomy in alert guinea pigs (Ris et al., 1995) and 6 months after bilateral transection of the vestibular nerve in alert rhesus monkeys (Waespe et al., 1992). Despite the potential for compensatory activity of the intact side in the unilateral labyrinthectomy studies (Kaufman et al., 1992; Ris et al., 1995), angular head rotation failed to influence expression of Fos in ipsilateral brainstem vestibular circuits (Kaufman et al., 1992) and did not modulate the firing rates of ipsilateral brainstem vestibular neurons (Ris et al., 1995). These data indicate that although unilateral surgical and arsanilate-induced labyrinthectomies disrupt rotation-induced changes in the activity of brainstem vestibular circuitry, brainstem vestibular nuclei remain intact. More importantly, Hunt et al. (1987) showed that bilateral intratympanic injections of sodium arsanilate in rats resulted in postural changes commensurate with changes observed after bilateral surgical labyrinthectomies. These behavioral changes included increased head dorsiflexion, flattened posture with limbs adducted, and an increased tendency to locomote backward and are indicative of vestibular dysfunction. Within $24 \mathrm{hr}$ after lesion, all rats in the present study demonstrated vestibular dysfunction and impaired contact righting. Arsanilate treatment did not interfere with the rat's ability to retrieve food pellets in the cylinder apparatus.

Testing paradigms. The influence of vestibular lesions on ATN HD cell activity was evaluated using two approaches. Using a within-subjects design for five rats, ATN electrode arrays were implanted, and HD cells were isolated and recorded before and after vestibular lesion. Before vestibular lesion these HD cells were recorded over at least four 8 min recording sessions. As described previously (Taube et al., 1990a; Taube, 1995), each HD cell was analyzed to determine: (1) preferred firing direction; (2) peak firing rate; (3) directional firing range; (4) mean background firing rate; (5) overall mean firing rate independent of HD; and (6) whether a $90^{\circ}$ rotation of the cue card resulted in a corresponding shift in the preferred firing direction of the HD cell. On completion of baseline data collection, the rat received bilateral intratympanic injections of sodium arsanilate. After recovery from the anesthetic $(\sim 30 \mathrm{~min})$, $\mathrm{HD}$ cell activity and the rat's vestibular function were monitored for $1 \mathrm{hr}$ after injection and then daily for at least 1 week after lesion. At the conclusion of postlesion analyses for a given HD cell, the electrode was advanced further through the ATN over the course of several months, and unit activity was screened for directional firing properties.

Using a between-subjects design for four additional rats, the vestibular system was lesioned by intratympanic sodium arsanilate administered at the time of the ATN electrode implantation. On recovery from surgery, unit activity was evaluated daily for HD cell firing properties over several months as the electrode array was advanced through the ATN. In addition, two other rats received bilateral intratympanic injections of $0.9 \%$ sterile saline $(0.1 \mathrm{ml} / \mathrm{side})$ at the time the ATN microelectrode array was surgically implanted. These final two rats served as intratympanic injection control subjects. Unit activity was screened for directional firing properties during daily screening ses- 
sions. Unit screening was terminated when the ATN electrode arrays had been advanced approximately $2 \mathrm{~mm}$.

Histology. At the conclusion of unit screening, rats were anesthetized with sodium pentobarbital (100 mg/kg, i.p.). Weak anodal current (15 $\mu \mathrm{A}$ for $10 \mathrm{sec}$ ) was passed through one of the electrode wires to mark the wire location by the deposition of iron (Prussian blue reaction). The rats were perfused transcardially with $0.9 \%$ saline followed by $10 \%$ formalin, and the brains were removed and placed into $10 \%$ formalin for at least $48 \mathrm{hr}$. The brains were then transferred to a $10 \%$ formalin solution containing $2 \%$ potassium ferrocyanide for $24 \mathrm{hr}$ and returned to a $10 \%$ formalin solution for $24 \mathrm{hr}$, after which the brains were placed into a $20 \%$ sucrose solution for $24 \mathrm{hr}$. The brains were then frozen, sectioned coronally at $30 \mu \mathrm{m}$, stained with cresyl violet, and examined under light microscopy to determine the location of recording sites. In addition, the anterior thalamic nuclei in the hemisphere opposite the electrode were assessed under light microscopy for potential gross damage resulting from the sodium arsanilate treatment, and no damage was observed. No attempt was made to histologically examine the consequences of sodium arsanilate treatment on the vestibular nuclei, eighth cranial nerve, or vestibular apparatus, because several previous reports have described such effects (Anniko and Wersäll, 1977; Chen et al., 1986; Kaufman et al., 1992).

\section{RESULTS}

Histological analyses after completion of unit screening verified that each electrode array had passed through the ATN in all 11 rats. Recording electrode tracks from all rats were identified by the Prussian blue reaction at the ventral-most limit of the anterodorsal nucleus of the anterior thalamus or immediately ventral to it. Because only one wire from each electrode was identified by the Prussian blue reaction, it is possible that for each rat a subset of electrode wires passed through the anteroventral nucleus of the anterior thalamus. With respect to the first five rats, eight HD cells were identified and recorded before vestibular lesion. A representative cell from each rat is illustrated in Figure 1. For the case illustrated in Figure $1 D$ a second HD cell was isolated on a separate wire and recorded; the data from this HD cell were included in all statistical analyses described below. In two other cases, Figure $1, B$ and $E$, second HD cells were simultaneously recorded on the same wire but could not be analyzed as separate cells. Therefore, the data from these two HD cells were not included in the statistical analyses described below. For each of the eight HD cells recorded before vestibular lesion, a $90^{\circ}$ rotation of the cue card of the cylinder led to a corresponding shift in the preferred firing direction of the cell. This finding and the values for peak firing rate and directional firing range were consistent with the discharge properties of ATN HD cells as described previously (Taube, 1995).

\section{Within-subject experiments}

At completion of baseline HD cell data collection, sodium arsanilate was administered under brevital anesthesia. Within $24 \mathrm{hr}$ after injection, bilateral intratympanic injections of sodium arsanilate produced behavioral sequelae characteristic of vestibular dysfunction: head dorsiflexion, postural abnormalities, increased horizontal locomotor behavior, and inhibition of contact-righting abilities (Horn et al., 1981; Ossenkopp et al., 1990). Arsanilate treatment did not interfere with the rat's ability to locomote throughout the cylinder and to retrieve food pellets.

In parallel with the behaviorally assessed vestibular dysfunction, the directional firing properties of each of the eight HD cells were abolished. In each case (five examples are illustrated in Fig. $1 A-E)$, the directional firing of ATN cells degraded over a time course of 1-24 hr postlesion. In all but one case (Fig. $1 A$ ), ATN cells continued to exhibit some degree of directional firing properties when assessed $1 \mathrm{hr}$ postlesion. Oscilloscope tracings of each
HD cell were stored, and representative photographs of waveforms were taken at each postlesion time point. Figure $1 F$ illustrates representative examples of the prevestibular and postvestibular lesion waveforms for an HD cell (the case depicted in Fig. $1 C$ ). Given the consistency of the prelesion and postlesion waveforms of each cell and the persistence of the same waveform across days, we are confident that electrical isolation of the same cell was maintained over the course of each experiment. Despite the fact that the vestibular lesion abolished the directional firing properties of the ATN cells, there was no significant change in overall mean firing rates $[t(5)=1.00 ; p>0.05$, not significant (NS)], when mean firing rates from $96 \mathrm{hr}$ postlesion recording sessions were compared with prelesion baseline mean firing rates. Although there was a greater than twofold increase in the background firing rates of the HD cells after lesion (Fig. 1), these rate increases did not reach significance $[t(5)=-1.94$, NS]. The lack of statistical significance probably reflects insufficient statistical power due to the small $n$. An increase in the background firing rates of the ATN cells after lesion was expected, because the overall mean firing rates did not change despite the loss of directionality. These results indicate that the vestibular apparatus is critical for the directional discharge of ATN neurons, although ATN neurons continue to fire in the absence of vestibular input.

\section{Influence of angular head velocity on ATN cell firing rates}

The discharge of ATN HD cells is modulated by angular head velocity (Taube, 1995; Blair and Sharp, 1995), in which the firing rate increases with faster head turns through the preferred firing direction. Angular velocity was determined using methods described previously (Taube, 1995). Briefly, angular head velocity was calculated for each $1 / 60$ sec sample by determining the HD for the given sample $(N)$ and the two samples that preceded and postceded it. This five sample episode was smoothed using the function: $n=\left(n_{t-2}+n_{t-1}+n+n_{t+1}+n_{t+2}\right) / 5$. The angular head velocity for this episode was taken as the slope of the best fit line through these five HD values and was then compared with the firing rate of that episode. The mean firing rates of ATN HD cells, recorded during both prelesion and $96 \mathrm{hr}$ postlesion sessions, were determined for specific angular head velocity intervals (0-90, $90-180,180-270$, and $270-1000^{\circ} / \mathrm{sec}$ ). Negative values for clockwise head turns were translated to absolute values and combined with the positive values (counterclockwise head turns) to determine the mean firing rates associated with each angular velocity interval. The prelesion and $96 \mathrm{hr}$ postlesion frequency distributions of these angular head velocities are illustrated in Figure $2 A$. A two-factor [angular velocity interval and time point (prelesion and $96 \mathrm{hr}$ postlesion)] repeated measures ANOVA on the angular velocity distribution data yielded a significant effect of angular velocity interval $\left[F_{(3,16)}=77.76 ; p<0.001\right]$ and a significant angular velocity interval $\times$ time point interaction $\left[F_{(3,16)}=4.79\right.$; $p<0.02]$, but no significant effect of time point $\left[F_{(1,16)}=3.11\right.$; NS]. These results indicate that: (1) the frequency distribution profile of overall head turns (greater number of slow head turns than fast turns) remained intact after lesion, as indicated by the significant angular velocity interval term; and (2) the total number of head turns exhibited before and $96 \mathrm{hr}$ postlesion were not different, as indicated by the absence of a significant time point term. However, Newman-Keuls multiple comparison tests revealed a significant decrease in the number of slow $\left(0-90^{\circ} / \mathrm{sec}\right)$ head turns exhibited at $96 \mathrm{hr}$ after sodium arsanilate treatment. The increase in the number of fast head turns (180-270 and 

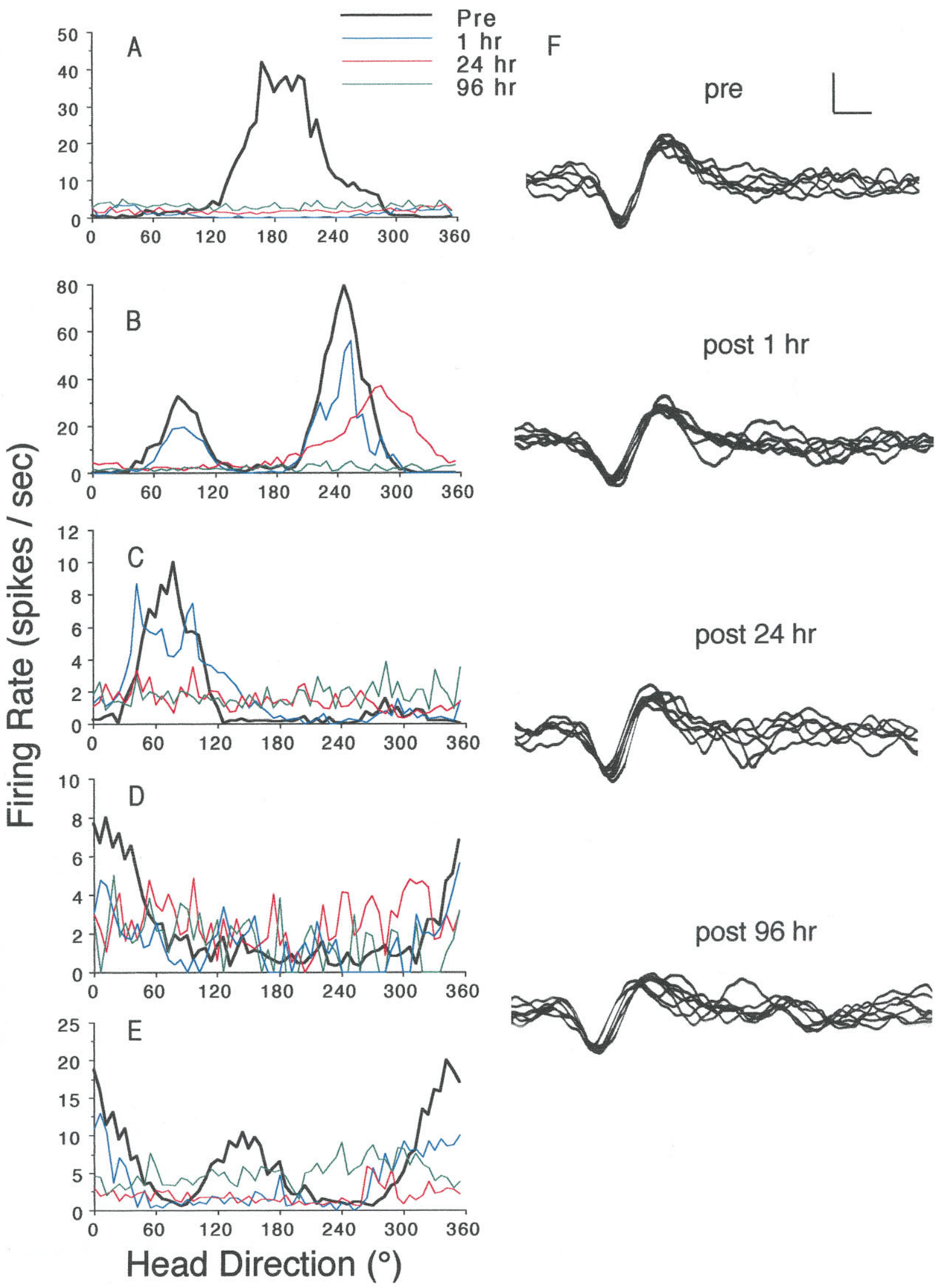

Figure 1. Time course of vestibular lesion-induced disruption of ATN HD cell firing properties. $A-E$, Firing rate versus HD tuning curves of five different ATN HD cells before and after intratympanic injection of sodium arsanilate. In each plot, the heavy black line (Pre) depicts the prelesion baseline activity of the cell. The remaining lines depict postlesion sampling times and are color coded as follows: blue, $1 \mathrm{hr}$; red, $24 \mathrm{hr}$; and green, $96 \mathrm{hr}$. For cases in $B-E$ the disruption of directional firing properties paralleled the onset of behavioral changes indicative of vestibular dysfunction. In $A$, at 1 hr postlesion, although the rat failed to exhibit a full disruption of vestibular function, the plot illustrates a loss of the directional firing of (Figure legend continues) 
A

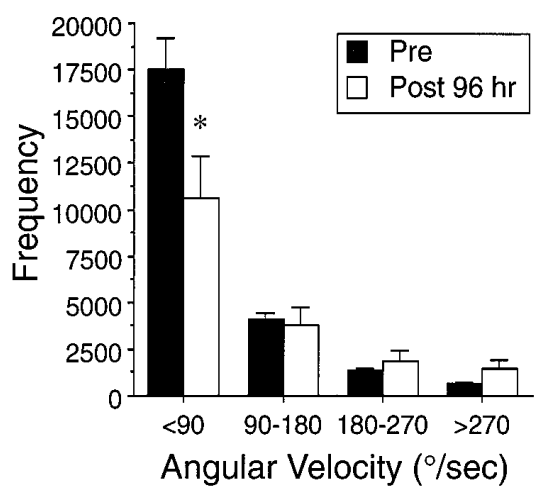

C

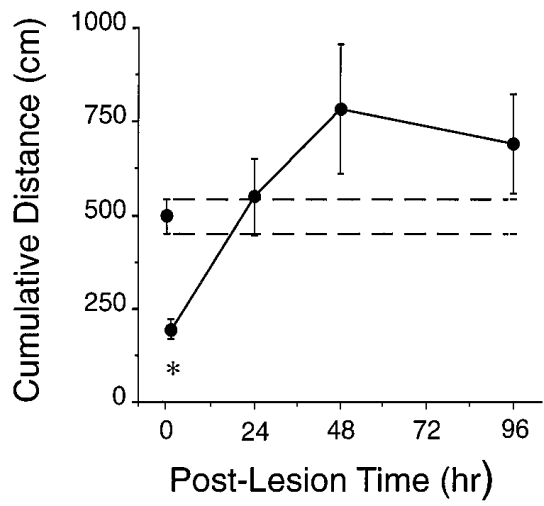

B

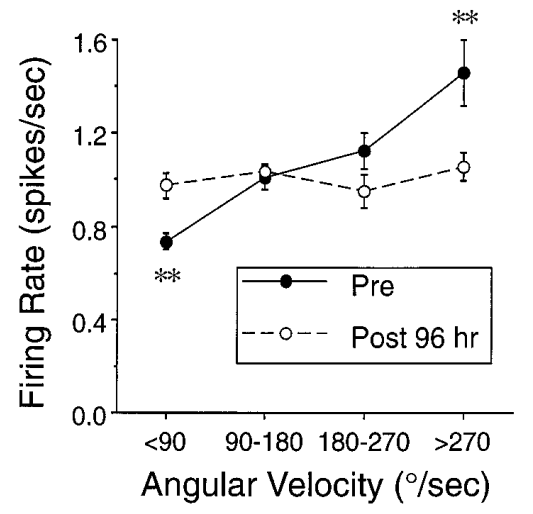

D

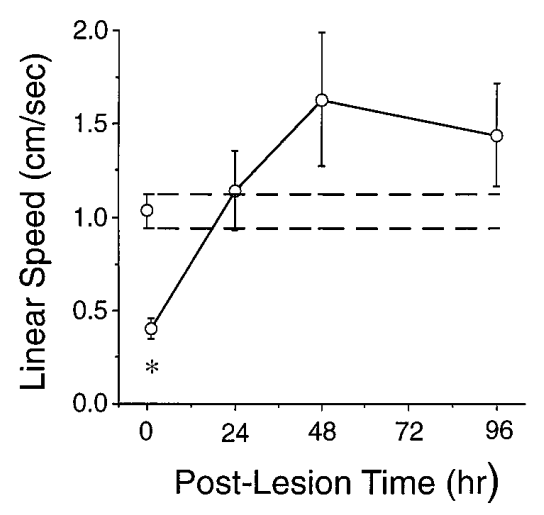

Figure 2. The influence of sodium arsanilate on angular and linear motor behavior. $A$, Intratympanic administration of sodium arsanilate induced a decrease in the number of slow head turns and an increase in the number of fast head turns. The absolute values of angular velocity were grouped into four intervals $\left(0-90,90-180,180-270\right.$, and $\left.270-1000^{\circ} / \mathrm{sec}\right)$. The graph depicts the mean + SEM frequency of head turns of each angular head velocity interval exhibited during prelesion ( filled bars) and $96 \mathrm{hr}$ postlesion (open bars) $8 \mathrm{~min}$ recording sessions. $B$, Influence of angular head velocity (in degrees per second) on firing rate of ATN HD cells during prelesion and $96 \mathrm{hr}$ postlesion recording sessions. The absolute values of angular velocity were grouped into the four intervals as above, and mean firing rates were determined for each interval. The graph depicts the mean normalized firing rate \pm SEM for all six cells, determined by dividing the firing rates of the cell for each angular velocity interval by the mean firing rate of that cell during that particular recording session. Prelesion baseline data are depicted by filled circles, whereas postlesion data (open circles) represent ATN unit activity recorded during the $96 \mathrm{hr}$ postlesion time point. Angular head velocity modulated the firing rates of HD cells recorded before, but not after, vestibular lesion. $C$, $D$, Influence of sodium arsanilate on locomotor activity. The graph depicts the mean \pm SEM cumulative distance traveled $(C)$ and the mean \pm SEM linear speed $(D)$. These measures were computed using the detected head LED samples from the video tracking system, collected during the 8 min recording session at each of the postlesion time points: 0 (prelesion baseline), 1, 24, 48, and $96 \mathrm{hr}$. For comparison, the dashed lines illustrate the values for the prelesion baseline data. Sodium arsanilate treatment induced a transient decrease in both the cumulative distance and linear speed of locomotor activity at $1 \mathrm{hr}$, followed by an increase at $48 \mathrm{hr}$, which recovered by $96 \mathrm{hr}$ postlesion. ${ }^{*} p<0.05$; ** $p<0.01$, compared with respective prelesion baseline data (Newman-Keuls tests).

$\left.270-1000^{\circ} / \mathrm{sec}\right)$ at $96 \mathrm{hr}$ postlesion, compared with the prelesion baseline, did not reach significance. Furthermore, because faster head turns have been reported to increase the firing rate of the HD cell at the preferred direction in control animals (Blair and Sharp, 1995; Taube, 1995), the higher incidence of faster head turns in lesioned rats would be expected to increase, not abolish, the firing rate of the cell after lesion. In sum, these results indicate that arsanilate-treated rats exhibited only mild differences in the total number and speed of their head turns and are unlikely to account for the complete disruption of direction-dependent discharge.

Angular head velocity modulated the firing rates of HD cells recorded before, but not after, vestibular lesion (Fig. $2 B$ ). That is, vestibular lesions also disrupted the influence of angular head velocity on the firing rates from all ATN HD cells as assessed after lesion. A two-factor [angular velocity interval and time point (prelesion and $96 \mathrm{hr}$ postlesion)] repeated measures ANOVA

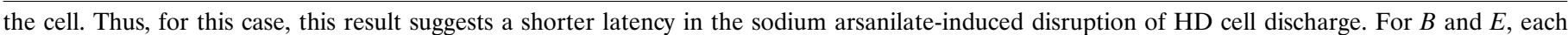

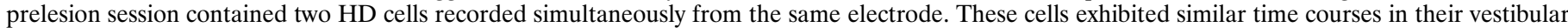

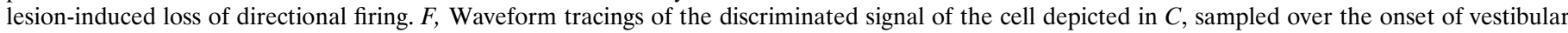

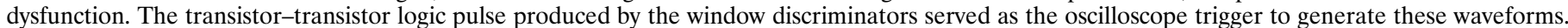

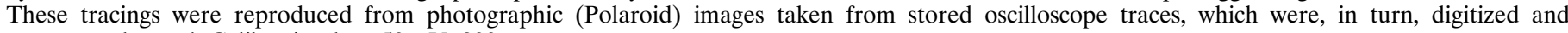
contrast-enhanced. Calibration bar, $50 \mu \mathrm{V}, 200 \mu \mathrm{sec}$. 

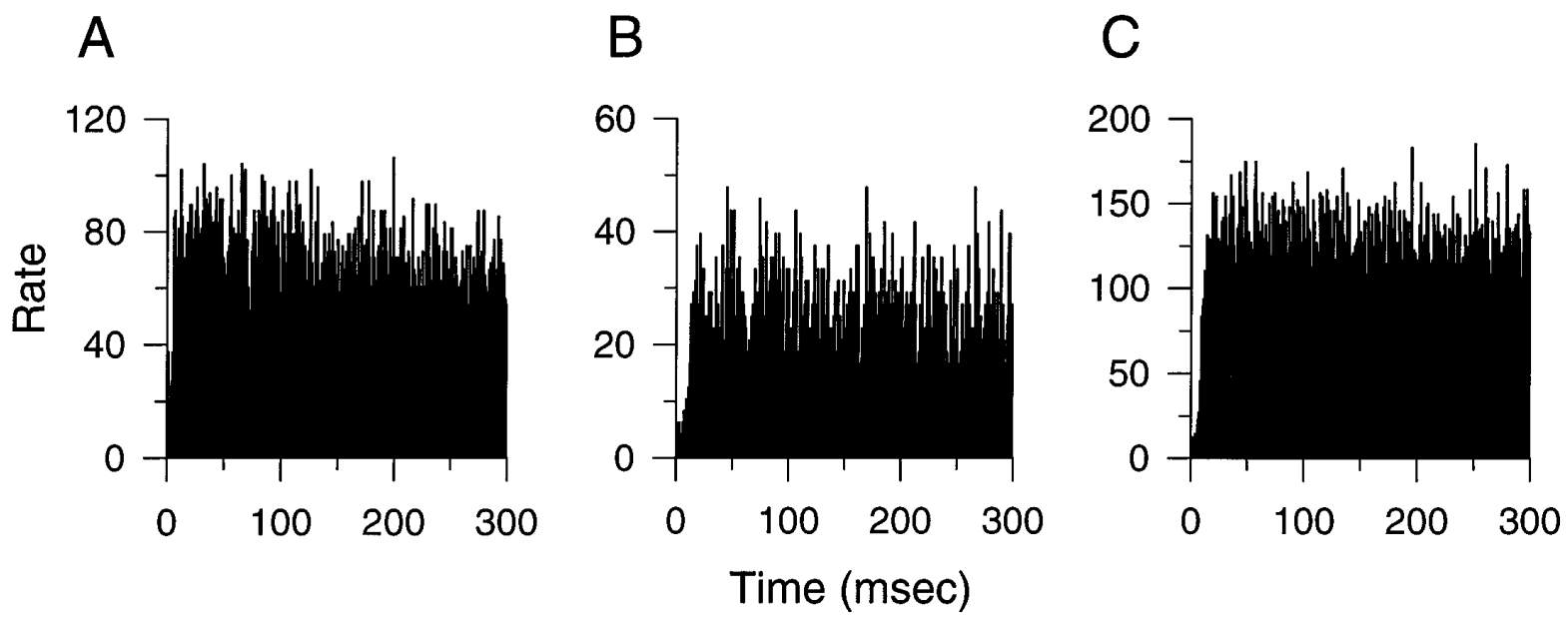

Figure 3. Representative example of an autocorrelation histogram for an ATN HD cell recorded before lesion $(A)$, an ATN HD cell recorded 96 hr postlesion $(B)$, and an ATN cell recorded after vestibular lesion that exhibited a burst pattern of firing $(C)$. These histograms were constructed by summing the number of times in which a spike occurred within each $1 \mathrm{msec}$ interspike interval from 0 to $300 \mathrm{msec}$, given the occurrence of a spike at time 0 . The ordinate of each graph represents the number of spikes that occurred in a $1 \mathrm{msec}$ interval during an 8 min session and expressed in hertz. None of the autocorrelation histograms revealed a complex spike firing pattern, nor did the functions indicate any particular rhythmicity to the spike train. In comparing the histograms for the HD cell before lesion $(A)$ with that of $96 \mathrm{hr}$ after lesion $(B)$, it seems that there is a modest increase in the interspike interval as a result of the lesion. The nondirectional burst cell shown in $C$ and in Figure $4 A$ has never been detected in the vestibular-intact rat; therefore, we have no information regarding the temporal patterns in the spike trains of this ATN cell type in the normal rat.

yielded a significant effect of angular velocity interval $\left[F_{(3,30)}=\right.$ $10.81 ; p<0.0001]$ and a significant angular velocity interval $\times$ time point interaction $\left[F_{(3,30)}=7.43 ; p<0.001\right]$, but no significant effect of time point $\left[F_{(1,10)}=1.73\right.$; NS]. Multiple comparisons tests revealed a significant difference between prelesion and postlesion measures at intervals of $<90$ and $>270^{\circ} / \mathrm{sec}$, as indicated in Figure $2 B$. These data indicate that prelesion, but not postlesion, ATN firing rates were modulated by angular head velocity. Thus, the loss of vestibular input to the ATN disrupted two functional correlates of HD cell firing: directionality and angular head velocity.

\section{Between-subject experiments}

Four rats received intratympanic sodium arsanilate injections at the time the ATN microelectrode array was surgically implanted. Sodium arsanilate treatment induced a permanent bilateral vestibular lesion, as assessed by loss of contact righting. After postoperative recovery, single-unit activity was monitored over several months, during which time the electrodes were advanced further through the ATN. No single units recorded in these rats exhibited HD firing properties, although histological analyses confirmed that the electrodes passed through the ATN. As an intratympanic injection control, two rats received intratympanic injections of $0.9 \%$ saline at the time the ATN microelectrode array was surgically implanted. Intratympanic saline did not disrupt vestibular function, because saline-treated rats continued to display contactrighting behavior over several months of unit screening and recording. In both saline control cases ATN HD cells were identified and recorded. The firing properties, waveforms, and HD by firing rate tuning curves for HD cells from saline-treated rats were comparable with those of intact rats (or with those recorded under baseline conditions, as illustrated in Fig. 1). Taken together with the data from our within-subject experiments, these results suggest that vestibular input is a critical signal for the discharge properties of ATN HD cells.

\section{Discharge properties of ATN neurons in vestibular-lesioned rats}

Although HD cells were not present in vestibular-lesioned rats, a subset of ATN neurons exhibited intermittent firing bursts that were unrelated to the rat's head direction (see Fig. $4 A$ ). These bursts of 20-40 spikes occurred on the order of two to five per minute. The discharge properties of these cells were neither related to the rats' $\mathrm{HD}$, nor were the spike bursts apparently temporally related to one another. The waveforms of this type of novel ATN signal were similar to the waveforms of ATN HD cells depicted in Figure $1 F$. From the nine sodium arsanilate-treated rats, we recorded 27 distinctly nondirectional firing ATN "burst cells" after lesion, as the rats retrieved food pellets in the cylinder. For each postlesion burst cell, autocorrelation histograms were plotted to provide an indication of whether this novel ATN unit exhibited a complex spike pattern of firing (Ranck, 1973) or whether the firing was modulated in a rhythmic manner (Barnes et al., 1990). Autocorrelation histograms were constructed by summing the number of times in which a spike occurred within each $1 \mathrm{msec}$ interspike interval bin from 0 to $300 \mathrm{msec}$, given the occurrence of a spike at time 0 . The sums were divided by the total time over which the cell was recorded (i.e., $8 \mathrm{~min}$ ) and expressed in hertz. The autocorrelation functions of the burst cells found in vestibular-lesioned rats were characterized by the frequent occurrence of spike bursts with short interspike intervals. None of the autocorrelation histograms for ATN burst cells suggested a complex spike firing pattern, nor did the functions indicate any particular rhythmicity to the spike train. An example of an autocorrelation histogram for one of the novel burst cells is presented in Figure $3 C$. For comparison purposes, autocorrelation histograms for an ATN HD cell recorded prelesion (Fig. 3A) and $96 \mathrm{hr}$ postlesion (Fig. 3B) are also depicted.

This type of ATN burst cell discharge was never observed in vestibular-intact rats. The firing properties of these burst ATN cells were also analyzed to determine whether the activity of the cell was influenced by the rat's angular head velocity. A one factor 
A

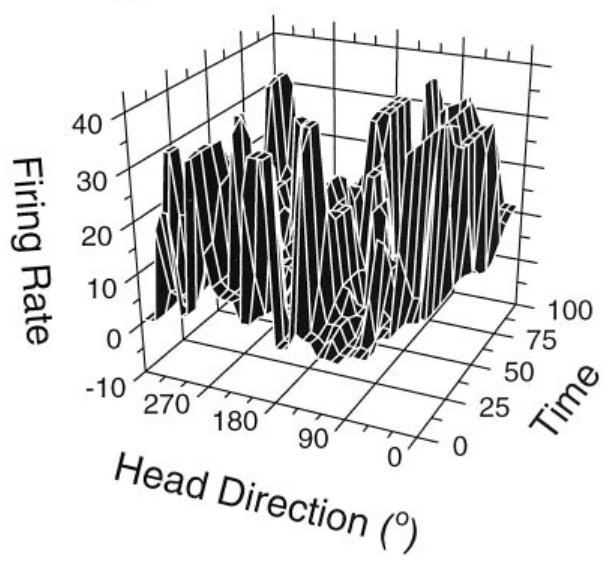

B

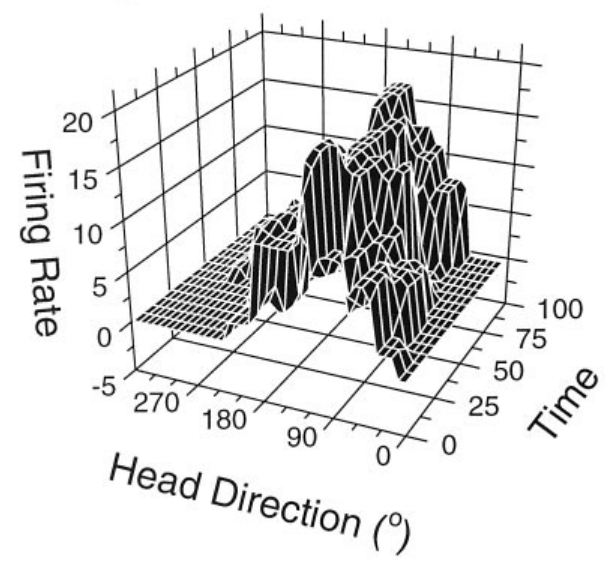

Figure 4. Three-dimensional plot of firing rate and head direction as a function of time in epochs of 5 sec duration of a distinctly nondirectional ATN burst cell recorded after vestibular lesion $(A)$ and an ATN HD cell recorded in the same rat before vestibular lesion $(B)$. The preferred firing direction of the HD cell depicted in $B$ is approximately $150^{\circ}$. For both plots, unit activity was recorded over 8 min sessions, during which the rat foraged in the cylindrical apparatus. These data were analyzed to derive an average HD (Batschelet, 1981) and firing rate in epochs of 5 sec duration. This figure provides an illustration of the HD cell discharge properties on a more realistic time scale. The postlesion burst cell depicted in $A$ exhibited a characteristic pattern of intermittent high firing rate events that were neither related to the rat's HD nor temporally organized. This ATN unit activity has not been observed in vestibular-intact rats and may represent a characteristic firing pattern that emerges as a consequence of the removal of vestibular inputs.

repeated measures ANOVA on the firing rates of the burst cells across the four angular velocity intervals yielded no significant modulatory effect of angular velocity interval $\left[F_{(3,78)}=1.35 ; \mathrm{NS}\right]$. Of the 27 burst-firing ATN single units recorded after lesion, 11 were recorded on electrode wires that had previously contained HD cells. Although it is possible that this novel cell type may represent the manner in which an HD cell fires in the absence of vestibular input, none of the five cells depicted in Figure 1 assumed this firing pattern during postlesion recording.

Given that vestibular signals are necessary for normal ATN HD cell firing and arguably for spatial orientation, it is possible that removal of vestibular input induces a perpetual drift in the preferred firing direction of the HD cell over time. Specifically, the vestibular input to the ATN may provide stability to the neural coding of current directional heading. In vestibular-deficient rats without such stability, the subset of active ATN neurons representing the allocentric heading may continually drift over time. This postlesion instability of the preferred firing direction of the cell would be consistent with navigational deficits observed in humans with vestibular dysfunction. To investigate this premise, the mean firing rate and mean HD (Batschelet, 1981) were determined in $5 \mathrm{sec}$ intervals for the 8 min recording session (Fig. $4 A$ ) for each burst cell. There was no pattern to the shift in the peak firing discharges of the cell with respect to HD over time; bursts seemed to occur at random directional headings. The example of this nondirectional cell can be compared with a clear example of the temporal firing properties of an ATN HD cell from a vestibular-intact rat, when firing rate and HD are analyzed in a similar manner (Fig. $4 B$ ). Although there is some variability in the moment-to-moment preferred firing direction of an ATN HD cell, there is little correlation between firing rate and HD in the distinctly nondirectional burst neurons recorded from vestibular-lesioned rats.

In sum, the characteristic pattern of ATN burst discharge was only observed in vestibular-lesioned rats and did not seem to originate from aberrant HD cells. Because the spatial and behavioral correlates of this cell type were not determined before the lesion, it is difficult to identify a distinct functional role for these cells. The novel ATN firing property found in the lesioned rats presumably reflects a compensatory change in the properties of the ATN or of the cortical and subcortical inputs to the ATN.

\section{Sodium arsanilate-induced behavioral changes}

The sodium arsanilate-induced postural changes, such as head dorsiflexion, that accompanied vestibular lesion did not disrupt the rats' ability to retrieve food pellets in the cylinder and are unlikely to contribute directly to the loss of ATN directional firing properties. Head dorsiflexion in a vestibular-intact rat does not disrupt HD cell firing properties, nor does it elicit HD cell firing. Qualitative assessments of HD cells have determined that neither head pitch (movement of the head up or down) nor roll (rotation of the head along the anteroposterior axis) failed to disrupt the firing of directional cells (Taube et al., 1990a). Lesioned rats exhibited a mild degree of behavioral recovery over a 3 month postlesion interval, but ATN directional firing properties never recovered. On completion of postlesion recordings ( $\sim 1$ week), the electrode arrays were advanced further through the ATN, and subsequent single-unit activity was evaluated for directional and spatial correlates. In each case, no single-unit recorded postlesion exhibited directional firing properties. In sum, ATN HD cells continued to discharge without vestibular input; however, their postlesion firing properties were distinctly nondirectional in nature.

Changes in locomotor behavior, as a consequence of sodium arsanilate treatment, may have contributed to the loss of directional firing in ATN neurons. Although this possibility seems unlikely, because HD cells in intact rats maintain their directionspecific discharge even when the animal is motionless (Taube et al., 1990a; Taube, 1995), we examined this premise by quantifying changes in locomotor behavior for each of the five within-subject rats. The cumulative distance traveled and the average speed of locomotion were determined by monitoring the detected position of the rat in $1 \mathrm{sec}$ sampling episodes. Values for cumulative distance (in centimeters) and average linear speed (centimeters per second) were calculated for each of five 8 min recording sessions (prelesion and 1, 24, 48, and $96 \mathrm{hr}$ postlesion) and are shown in Figure 2, $C$ and $D$, respectively. ANOVAs revealed a 
significant repeated measures effect for cumulative distance $\left[F_{(4,16)}=9.26 ; p<0.001\right]$ and for average speed $\left[F_{(4,16)}=9.27\right.$; $p<0.001]$. Post hoc Newman-Keuls multiple comparison tests revealed a significant decrease in both distance traveled and speed at the $1 \mathrm{hr}$ postlesion time point compared with prelesion baseline measures. At $48 \mathrm{hr}$ postlesion rats exhibited a moderate, but nonsignificant increase in both locomotor measures. There were no differences in either locomotor measure at the 24 and $96 \mathrm{hr}$ postlesion time points compared with baseline values. These data indicate that sodium arsanilate-induced vestibular dysfunction was accompanied by a transient decline in locomotor behavior at $1 \mathrm{hr}$ postlesion followed by a slight increase at $48 \mathrm{hr}$ postlesion, which returned to near baseline levels by $96 \mathrm{hr}$ postlesion. Although the vestibular lesion-induced alterations in locomotor behavior may have contributed to the initial disruption of ATN directional firing, the present data suggest that the lesion-induced changes in motor behavior were transient and, therefore, cannot explain the absence of ATN HD cells in rats recorded several months postlesion.

The increase in cumulative distance traveled at $48 \mathrm{hr}$ postlesion is consistent with previous findings of increased horizontal locomotor activity after sodium arsanilate treatment (Ossenkopp et al., 1990). It is interesting to note that despite the significant decline in motor behavior at $1 \mathrm{hr}$ postlesion, in four of the five examples (Fig. $1 B-E$ ), ATN HD cells continued to exhibit some degree of direction-specific discharge. These findings are also consistent with previous studies showing that HD cell discharge is largely independent of the rat's locomotor behavior, although some modulation of firing rate has been reported based on the rat's linear head velocity (Taube et al., 1990a; Taube, 1995). As with angular head velocity, HD cell firing rates are positively correlated with the animal's linear velocity. Thus, higher firing rates are associated with a faster speed of linear movement. Given this relationship, a manipulation that increases locomotor behavior might be expected to augment HD cell firing, rather than result in the observed abolition of such firing. Taken together, although we cannot exclude the possibility that patterns of aberrant locomotor behavior in vestibular-lesioned rats may contribute to the disruption of the ATN HD cell signal, we consider this possibility unlikely, because: (1) locomotor behavior is not the primary determinant of HD cell discharge in intact animals; (2) the overall changes in locomotor behavior observed in lesioned rats were moderate and only involved the frequency distribution of speeds; and (3) given the established relationships between HD cell firing and locomotor behavior, the changes we observed in both linear and angular locomotor behavior were frequently not in the direction we expected. That is, the decline in HD cell firing was associated with increased angular and linear motion.

Finally, the intratympanic administration of sodium arsanilate also disrupted the auditory capabilities of the treated rats, because observations of these rats suggested a diminished sensitivity to auditory stimuli. Although it is possible that such hearing loss contributed to the disruption of the directional firing properties of ATN neurons, we consider this possibility unlikely for two reasons. First, disruption of the tympanic membrane alone, as in the intratympanic saline-treated rats, failed to interfere with ATN HD cell activity. Second, visual cues have been shown to exert more influence over HD cell firing than auditory cues (Goodridge et al., 1995), and the visual cues remained present throughout the recording periods after the chemical labyrinthectomies.

\section{DISCUSSION}

The present results demonstrate that removal of ATN vestibular input disrupts the directional firing of ATN neurons. ATN single units recorded from vestibular-deficient rats failed to exhibit directional firing properties. A subset of cells recorded from vestibular-lesioned rats exhibited a novel discharge pattern characterized by intermittent bursts of activity that were not apparently related to the rat's HD. In addition to abolishing ATN directional firing, vestibular lesions disrupted the modulatory influence of angular head velocity on the firing rate of ATN neurons. Thus, vestibular information is critical for the normal directional discharge of ATN HD cells.

The vestibular system enables the detection of head position and head motion in space. In combination with the visual landmark system and other internal cues defined above, these systems contribute information that supports spatial orientation and navigation (Barlow, 1964; Beritoff, 1965; Gallistel, 1990; Pozzo et al., 1991; Brookes et al., 1993; McNaughton et al., 1995). Presumably, the directional sense is computed from a neural system that integrates current information from the two navigational systems described above. HD cells are thought to encode the animal's momentary directional heading in absolute space. Vestibular stimulation presented above the vestibular threshold influences the preferred firing direction of HD cells (Blair and Sharp, 1996). In the present experiments, vestibular lesions resulted in a complete loss of the directional firing of ATN neurons. In addition to the PoS, HD cells have been identified in the lateral dorsal nucleus of thalamus (Mizumori and Williams, 1993); the striatum (Wiener, 1993); the retrosplenial and medial prestriate cortex (Chen et al., 1994); and the lateral mammillary nuclei (Leonhard et al., 1996). Furthermore, in rats, the PoS is reciprocally connected with the ATN, the lateral dorsal thalamic nucleus, and, to a lesser degree, the retrosplenial cortex (Swanson and Cowan, 1977; van Groen and Wyss, 1990, 1992, 1995; Shibata, 1993). The organization of a central representation of directional bearing undoubtedly requires the coordinated integration of neural signals from several brain areas. Nonetheless, with respect to the ATN HD cells, it seems that vestibular information is critical. Whether a vestibular lesion disrupts directional activity in other brain areas containing HD cells remains to be determined. HD cell firing is abolished in the PoS after neurotoxic ATN lesions (Goodridge and Taube, 1993), suggesting that the HD cell signal may be generated in the ATN and conveyed to the PoS. Given that vestibular lesions abolish the directional firing of ATN neurons, and ATN lesions disrupt PoS HD cell activity, these results suggest that PoS HD activity may also be disrupted after vestibular lesion.

The firing properties of HD cells are maintained in the absence of the cylinder cue card (Taube et al., 1990b; Goodridge and Taube, 1995) and in blindfolded animals (Goodridge et al., 1995), suggesting that internal cues may provide sufficient information to support HD cell firing. Goodridge and Taube (1995) reported that if the removed cue card was returned to the cylinder in a position that conflicted with the current firing orientation of the cell, the HD cell promptly shifted its preferred firing direction to the originally established relationship with the landmark cue. This result was taken as evidence that internal sensory sources can support HD cell firing, although visual landmark cues are used preferentially over internal sensory cues. Furthermore, it has been hypothesized that the integration of angular velocity information from 
the vestibular system is necessary for the maintenance of an internal representation of directional bearing in the absence of familiar landmark cues (McNaughton et al., 1991, 1995). Consequently, it is interesting that the loss of vestibular function abolished the directional firing of ATN neurons even in a familiar environment in the presence of landmarks previously shown to influence HD cells. This result indicates that vestibular information is not only relevant under conditions in which path integration is necessary, but is also essential for the generation of the HD cell signal. Presumably, during navigation, animals continually monitor internal cue systems, and this information is corrected by periodic reference to landmark cues (Barlow, 1964; Gallistel, 1990). This view necessitates a critical role for internal cue systems in spatial navigation and in the maintenance of central representations of directional heading. The present findings therefore suggest a potential neurobiological mechanism that accounts for the spatial navigational deficits observed in vestibular-lesioned humans (Beritoff, 1965; Heimbrand et al., 1991; Pozzo et al., 1991; Brookes et al., 1993) and laboratory animals (Potegal et al., 1977; Miller et al., 1983; Matthews et al., 1989; Ossenkopp and Hargreaves, 1993).

Because HD cells encode the relationship between head position and environmental cues, the activity of these cells is likely to be influenced by inputs from the vestibular system. Anatomical studies have defined the cortical projections of the vestibular nuclei in the cat and primate. The vestibular nuclei project primarily to the medial geniculate nucleus and the ventral posterior inferior thalamic nucleus (Abraham et al., 1977; Lang et al., 1979). These thalamic areas project to the parietoinsular vestibular cortex in nonhuman primates (Büttner and Lang, 1979; Grüsser et al., 1990). Vestibular information could then reach the ATN and/or PoS by way of vestibular cortex projections to the retrosplenial cortex. Alternatively, a second pathway exists whereby the ATN may receive vestibular information (Taube et al., 1996a). The rat medial vestibular nuclei, which encode horizontal semicircular canal information, project directly to the dorsal tegmental nucleus (Liu et al., 1984). The dorsal tegmental nucleus innervates the lateral mammillary nuclei (Allen and Hopkins, 1989), which in turn send a major projection to the ATN (Shibata, 1992). The present data illustrate the critical involvement of vestibular input in the ATN neural coding of direction. The pathway outlined above represents a more direct route by which vestibular information could influence the physiological output of the ATN.

It is noteworthy that intratympanic sodium arsanilate led to the degeneration of the vestibular nerve in rats (Chen et al., 1986) and that the spontaneous activity of central vestibular neurons recovers after bilateral vestibular nerve transection (Waespe et al., 1992) or unilateral surgical labyrinthectomy (Ris et al., 1995). Furthermore, Ris et al. (1995) reported that despite compensatory changes after unilateral labyrinthectomy, angular head rotation failed to modulate brainstem vestibular neuron firing rates. In light of these findings it is surprising that arsanilate-induced labyrinthectomy disrupted the ATN HD cell signal. Indeed, taken together, these findings imply that the loss of directional activity in the ATN after chemical labyrinthectomy is unlikely attributed to the absence of a tonic discharge signal from the vestibular nuclei. Rather, it is the loss of modulation in the vestibular signal that seems to culminate in the disruption of the directional signal.

Interestingly, $\mathrm{HD}$ cell firing in the PoS encodes the rat's past HD, whereas ATN HD cell firing encodes the future HD by approximately $25 \mathrm{msec}$ (Blair and Sharp, 1995; Taube and Muller, 1995). Blair and Sharp (1995) proposed that anticipatory ATN HD cell firing is accomplished by a thalamocortical circuit that integrates current HD and angular head motion at the level of the ATN. It is possible that vestibular information, encoded as angular head motion, is projected to the ATN via pathways through dorsal tegmental and lateral mammillary nuclei. That ATN cells exhibit anticipatory HD firing is consistent with preliminary data indicating that ATN lesions disrupt the directional firing of PoS cells (Goodridge and Taube, 1993). Although vestibular inputs apparently play a critical role in the generation of the ATN HD cell signal, it is likely that other internal cue systems are also involved in the organization of the HD cell signal (for discussion of this point, see Taube et al., 1996a). For example, mild restraint procedures interfere with the location-specific firing of hippocampal pyramidal cells (Foster et al., 1989) and disrupt the firing properties of ATN HD cells (Taube, 1995). Similarly, when rats are passively transported from a familiar to an unfamiliar environment aboard a wheeled cart, ATN HD cells exhibit a marked shift in their preferred firing directions (Taube et al., 1996b). Under conditions in which the rat walks into the novel environment, these directional shifts are not observed (Taube and Burton, 1995). These results are consistent with similar findings from the human literature (Rieser et al., 1995) and provide support for the notion that motor efference copy cues and proprioceptive cues also influence neural coding of allocentric space. Given these findings, it is conceivable that arsanilate-induced alterations in locomotor activity might result in aberrant motor signals, which in turn indirectly contribute to the disruption of ATN directional cell firing.

Lesions of the vestibular system abolish the directional firing properties of ATN neurons. The present results provide direct neurobiological evidence that the vestibular system is critical for the central representation of direction. These data have important implications for current theories and neural network modeling of spatial cognition, because several models have hypothesized a critical role for the vestibular system (McNaughton et al., 1991, 1995; Touretzky and Redish, 1996) or angular head motion (Blair and Sharp, 1995) in the generation of the HD signal. Because HD cells are a neurophysiological correlate of a sense of direction, the present findings suggest that the vestibular system is a necessary neurobiological substrate for accurate spatial cognition.

\section{REFERENCES}

Abraham L, Copack PB, Gilman S (1977) Brain stem pathways for vestibular projections to cerebral cortex in the cat. Exp Neurol 55:436-448. Allen GV, Hopkins DA (1989) Mamillary body in the rat: topography and synaptology of projections from the subicular complex, prefrontal cortex, and midbrain tegmentum. J Comp Neurol 286:311-336.

Anniko M, Wersäll J (1977) Experimentally (atoxyl) induced ampullar degeneration and damage to the maculae utriculi. Acta Otolaryngol 83:429-440.

Barlow JS (1964) Inertial navigation as a basis for animal navigation. J Theor Biol 6:76-117.

Barnes CA, McNaughton BL, Mizumori SJY, Leonard BW, Lin L-H (1990) Comparison of spatial and temporal characteristics of neuronal activity in sequential stages of hippocampal processing. Prog Brain Res 83:287-300.

Batschelet E (1981) Circular statistics in biology. New York: Academic. Beritoff JS (1965) Neural mechanisms of higher vertebrate behavior. Boston: Little, Brown.

Berthoz A, Isräel I, Georges-François P, Grasso R, Tsuzuku T (1995) Spatial memory of body linear displacement: what is being stored? Science 269:95-98.

Blair HT, Sharp PE (1995) Anticipatory head direction signals in ante- 
rior thalamus: evidence for a thalamocortical circuit that integrates angular head motion to compute head direction. $\mathrm{J}$ Neurosci 15:6260-6270.

Blair HT, Sharp PE (1996) Visual and vestibular influences on headdirection cells in the anterior thalamus of the rat. Behav Neurosci 110:643-660.

Brookes GB, Gresty MA, Nakamura T, Metcalfe T (1993) Sensing and controlling rotational orientation in normal subjects and patients with loss of labyrinthine function. Am J Otol 14:349-351.

Büttner U, Lang W (1979) The vestibulocortical pathway: neurophysiological and anatomical studies in the monkey. Prog Brain Res 50:581-588.

Chen LL, Lin LH, Green EJ, Barnes CA, McNaughton BL (1994) Headdirection cells in the rat posterior cortex. I. Anatomical distribution and behavioral modulation. Exp Brain Res 101:8-23.

Chen Y-C, Pellis SM, Sirkin DW, Potegal M, Teitelbaum P (1986) Bandage backfall: labyrinthine and non-labyrinthine components. Physiol Behav 37:805-814.

Foster TC, Castro CA, McNaughton BL (1989) Spatial selectivity of rat hippocampal neurons: dependence on preparedness for movement. Science 244:1580-1582.

Gallistel CR (1990) The organization of learning. Cambridge, MA: MIT.

Goodridge JP, Taube JS (1993) Lesions of the anterior thalamic nucleus disrupt head direction cell firing in the dorsal presubiculum. Soc Neurosci Abstr 19:796.

Goodridge JP, Taube JS (1995) Preferential use of the landmark navigational system by head direction cells in rats. Behav Neurosci 109:49-61.

Goodridge JP, Worboys KA, Dudchenko P, Taube JS (1995) The response of head direction cells to non-visual cues. Soc Neurosci Abstr 21:945.

Grüsser OJ, Pause M, Schreiter U (1990) Localisation and responses of neurones in the parieto-insular vestibular cortex of awake monkeys (Macaca fascicularis). J Physiol (Lond) 430:537-557.

Heimbrand S, Muller M, Schweigart G, Mergner T (1991) Perception of horizontal head and trunk rotation in patients with loss of vestibular functions. J Vestib Res 1:291-298.

Horn KM, DeWitt JR, Nielson HC (1981) Behavioral assessment of sodium arsanilate induced vestibular dysfunction in rats. Physiol Psychol 9:371-378.

Hunt MA, Miller SW, Nielson HC, Horn KM (1987) Intratympanic injection of sodium arsanilate (Atoxyl) solution results in postural changes consistent with changes described for labyrinthectomized rats. Behav Neurosci 101:427-428.

Kaufman GD, Anderson JH, Beitz AJ (1992) Fos-defined activity in rat brainstem following centripetal acceleration. J Neurosci 12:4489-4500.

Kubie JL (1984) A driveable bundle of microwires for collecting singleunit data from freely-moving rats. Physiol Behav 32:115-118.

Lang W, Büttner-Ennever JA, Büttner U (1979) Vestibular projections to the monkey thalamus: an autoradiographic study. Brain Res 177:3-17.

Leonhard CM, Stackman RW, Taube JS (1996) Head direction cells recorded from the lateral mammillary nuclei in rats. Soc Neurosci Abstr 22:1873

Liu R, Chang L, Wickern G (1984) The dorsal tegmental nucleus: an axoplasmic transport study. Brain Res 310:123-132.

Matthews BL, Ryu JH, Bockaneck C (1989) Vestibular contribution to spatial orientation: evidence of vestibular navigation in an animal model. Acta Otolaryngol 468:149-154.

McNaughton BL, Chen LL, Markus EJ (1991) "Dead reckoning," landmark learning, and the sense of direction: a neurophysiological and computational hypothesis. J Cognit Neurosci 3:190-202.

McNaughton BL, Knierim JJ, Wilson MA (1995) Vector encoding and the vestibular foundations of spatial cognition: neurophysiological and computational mechanisms. In: The cognitive neurosciences (Gazzaniga $\mathrm{M}$, ed), pp 585-595. Boston: MIT.

Miller S, Potegal M, Abraham L (1983) Vestibular involvement in a passive transport and return task. Physiol Psychol 11:1-10.

Mittelstaedt M-L, Mittelstaedt H (1980) Homing by path integration in a mammal. Naturwissenschaften 67:566-567.

Mizumori SJY, Williams JD (1993) Directionally selective mnemonic properties of neurons in the lateral dorsal nucleus of the thalamus of rats. J Neurosci 13:4015-4028.

Ossenkopp K-P, Hargreaves EL (1993) Spatial learning in an enclosed eight-arm radial maze in rats with sodium arsanilate-induced labyrinthectomies. Behav Neural Biol 59:253-257.
Ossenkopp K-P, Prkacin A, Hargreaves EL (1990) Sodium arsanilateinduced vestibular dysfunction in rats: effects on open-field behavior and spontaneous activity in the automated digiscan monitoring system. Pharmacol Biochem Behav 36:875-881.

Paxinos G, Watson C (1986) The rat brain in stereotaxic coordinates, Ed 2. New York: Academic.

Potegal M, Day MJ, Abraham L (1977) Maze orientation, visual and vestibular cues in two-maze spontaneous alternation of rats. Physiol Psychol 5:414-420.

Pozzo T, Berthoz A, LeFort L, Vitte E (1991) Head stabilization during various locomotor tasks in humans. II. Patients with bilateral peripheral vestibular deficits. Exp Brain Res 85:208-217.

Ranck Jr JB (1973) Studies on single neurons in dorsal hippocampal formation and septum in unrestrained rats. I. Behavioral correlates and firing repertoires. Exp Neurol 41:461-535.

Rieser JJ, Pick Jr HL, Ashmead DH, Garing AE (1995) Calibration of human locomotion and models of perceptual-motor organization. J Exp Psychol Hum Percept Perform 21:480-497.

Ris L, de Waele C, Serafin M, Vidal P-P, Godaux E (1995) Neuronal activity in the ipsilateral vestibular nucleus following unilateral labyrinthectomy in the alert guinea pig. J Neurophysiol 74:2087-2099.

Sharp PE, Blair HT, Tzanetos DB (1995) Influences of vestibular and visual motion information on the spatial firing patterns of hippocampal place cells. J Neurosci 15:173-189.

Shibata H (1992) Topographic organization of subcortical projections to the anterior thalamic nuclei in the rat. J Comp Neurol 323:117-127.

Shibata H (1993) Direct projections from the anterior thalamic nuclei to the retrohippocampal region in the rat. J Comp Neurol 337:431-445.

Shoham S, Chen Y-C, DeVietti TL, Teitelbaum P (1989) Deafferentation of the vestibular organ: effects on atropine-resistant EEG in rats. Psychobiology 17:307-314.

Swanson LW, Cowan WM (1977) An autoradiographic study of the organization of the efferent connections of the hippocampal formation in the rat. J Comp Neurol 172:49-84.

Taube JS (1995) Head direction cells recorded in the anterior thalamic nuclei of freely moving rats. J Neurosci 15:70-86.

Taube JS, Burton HL (1995) Head direction cell activity monitored in a novel environment and during a cue conflict situation. J Neurophysiol 74:1953-1971.

Taube JS, Muller RU (1995) Head direction cell activity in the anterior thalamic nuclei, but not the postsubiculum, predicts the animal's future directional heading. Soc Neurosci Abstr 21:946.

Taube JS, Muller RU, Ranck JB (1990a) Head direction cells recorded from the postsubiculum in freely moving rats. I. Description and quantitative analysis. J Neurosci 10:420-435.

Taube JS, Muller RU, Ranck JB (1990b) Head direction cells recorded from the postsubiculum in freely moving rats. II. The effects of environmental manipulations. J Neurosci 10:436-447.

Taube JS, Goodridge JP, Golob EJ, Dudchenko PA, Stackman RW (1996a) Processing the head direction cell signal: a review and commentary. Brain Res Bull 40:477-486.

Taube JS, Stackman RW, Dudchenko PA (1996b) Head-direction cell activity monitored following passive transport into a novel environment. Soc Neurosci Abstr 22:1873.

Touretzky DS, Redish AD (1996) Theory of rodent navigation based on interacting representations of space. Hippocampus 6:247-270.

van Groen T, Wyss JM (1990) The postsubicular cortex in the rat: characterization of the fourth region of the subicular cortex and its connections. Brain Res 529:165-177.

van Groen T, Wyss JM (1992) Projections from the laterodorsal nucleus of the thalamus to the limbic and visual cortices in the rat. J Comp Neurol 324:427-448.

van Groen T, Wyss JM (1995) Projections from the anterodorsal and anteroventral nucleus of the thalamus to the limbic cortex in the rat. J Comp Neurol 358:584-604.

Waespe W, Schwarz U, Wolfensberger M (1992) Firing characteristics of vestibular nuclei neurons in the alert monkey after bilateral vestibular neurectomy. Exp Brain Res 89:311-322.

Wiener SI (1993) Spatial and behavioral correlates of striatal neurons in rats performing a self-initiated navigational task. J Neurosci 13:3802-3817.

Wiener SI, Korshunov VA, Garcia R, Berthoz A (1995) Inertial, substratal and landmark cue control of hippocampal CA1 place cell activity. Eur J Neurosci 7:2206-2219. 